${ }^{1}$ Universidade Estadual de Campinas, Faculdade de Odontologia de Piracicaba, Departamento de Diagnóstico Oral, Laboratório de Microbiologia e Imunologia, Piracicaba, São Paulo, Brasil.

${ }^{2}$ Universidade José do Rosário Vellano, Faculdade de Medicina, Laboratório de Farmacogenética e Biologia Molecular \& Centro de Pesquisa em Ciência Animal, Departamento de Patologia e Farmacologia Animal, Alfenas, Minas Gerais, Brasil.

*Corresponding author: Marcelo F. G. Boriollo, Universidade Estadual de Campinas, Faculdade de Odontologia de Piracicaba, Departamento de Diagnóstico Oral, Laboratório de Microbiologia e Imunologia, Avenida Limeira, 901 - CEP 13414-903, Piracicaba, São Paulo, Brasil. Telephone / Fax: +55 019 21065250 / 2106-5251 / 2106-5355 E-mail marcelofgb@yahoo.com.br

Received: May 25, 2017

Accepted: August 09, 2017

\section{Isoenzyme genotyping and phylogenetic analysis of oxacillin-resistance Staphylococcus aureus isolates}

\author{
Marcelo Fabiano Gomes Boriollo ${ }^{1,2, *}$, Manoel Francisco \\ Rodrigues Netto ${ }^{1,2}$, Jeferson Júnior da Silva ${ }^{1}$, \\ Thaísla Andrielle da Silva², Maysa Eduarda de Castro², \\ Júlio César Elias ${ }^{2}$ and José Francisco Höfling ${ }^{1}$
}

Aim: The propagation of $S$. aureus in hospital and dental environments is considered an important public health problem since resistant strains can cause serious infections in humans. The genetic variability of 99 oxacillin-resistant $S$. aureus isolates (ORSA) from the dental patients (oral cavity) and environments (air) was studied by isoenzyme genotyping. Methods: S. aureus isolates were studied using isoenzyme markers (alcohol dehydrogenase, sorbitol dehydrogenase, mannitol-1-phosphate dehydrogenase, malate dehydrogenase, glucose dehydrogenase, D-galactose dehydrogenase, glucose-6-phosphate dehydrogenase, catalase and $\alpha / \beta$-esterase) and genetic (Nei's statistics) and cluster analysis (UPGMA algorithm). Results: A highly frequent polyclonal pattern was observed in this population of ORSA isolates, suggesting various sources of contamination or microbial dispersion. Genetic relationship analysis showed a high degree of polymorphism between the strains, and it revealed three taxa (A, B and $C$ ) distantly genetically related $\left(0.653 \leq d_{\mathrm{ij}} \leq 1.432\right)$ and fifteen clusters (I to XV) moderately related $\left(0.282 \leq d_{i j}<0.653\right)$. These clusters harbored two or more highly related strains $\left(0 \leq d_{i j}<0.282\right)$, and the existence of microevolutionary processes in the population of ORSA. Conclusion: This research reinforces the hypothesis of the existence of several sources of contamination and/or dispersal of ORSA of clinical and epidemiologically importance, which could be associated with carriers (patients) and dental environmental (air).

Keywords: Oxacillin-resistant Staphylococcus aureus. Genetic variability. Propagation dynamics. MLEE. Clustering analysis. 


\section{Introduction}

The dissemination of $S$. aureus is considered an important public health problem because resistant strains can cause serious infections, especially in children and hospital patients ${ }^{1-3}$. Dentists treat a wide variety of patients, a fact that exposes these health professionals to people colonized or infected with resistant microorganisms ${ }^{2,4}$. High rates of resistance to antibiotics used during odontological prophylaxis have been detected in pathogens associated with bacterial endocarditis, for example, S. aureus ${ }^{5-8}$. Strains of $S$. aureus can be disseminated during dental treatment and occasionally lead to the contamination and infection of patients and dentists. Certain aspects of odontological practice can contribute to the dissemination of microorganisms 9,10 . The skin, environment and instruments can be contaminated with saliva, blood or debris during routine odontological treatment ${ }^{10,11}$. Several researchers have noted an increase in the amount of microorganisms present during clinical procedures in odontological environments, suggesting contamination from aerosols, especially when high-speed devices or ultrasonic scalers are used ${ }^{12,13}$. Among the species identified in microbiological studies, streptococci of the group viridans and Staphylococcus spp. are the most prevalent microorganisms found on surfaces of odontological equipment ${ }^{12-15}$, including methicillin-resistant $S$. aureus, which has been detected on odontological operatory surfaces, air-water syringes and recliner chairs ${ }^{16}$. Additionally, bacteria and fungi were significantly more frequent in dentist's hand with rings than those without rings, being Staphylococcus aureus, Escherichia coli and Candida albicans highly prevalent among the isolated potentially pathogenic microorganisms ${ }^{10}$.

Phenotypic methods (biotyping, serotyping, bacteriophage or bacteriocin typing and antimicrobial susceptibility profiles) and genotypic [pulsed-field gel electrophoresis (PFGE) and other methods based on the restriction of genomes, analysis of plasmids, typing methods based on polymerase chain reaction (PCR)] of microbiological characterization have elucidated the relationship and the distribution of human pathogens, which is considered essentially important for the epidemiology and control of hospital infections ${ }^{17}$. Isoenzymatic typing [multilocus enzyme electrophoresis (MLEE)] has been used for several decades as a "gold standard" in population genetics studies of eukaryotes ${ }^{18-20}$ and systematic studies ${ }^{21}$, as well as in large-scale studies for determining the genetic diversity and structure of natural populations of a variety of bacteria species ${ }^{22-24}$ and fungi ${ }^{25-27}$. This method represents an invaluable complement to the more recently developed molecular typing methods, particularly for largescale epidemiological studies ${ }^{28}$. In addition, MLEE possesses excellent typability (i.e., the percentage of different strains obtained) and reproducibility (i.e., the percentage of strains that display the same results in repetitive tests) and is associated with great discriminatory power (i.e., the ability to differentiate unrelated strains) ${ }^{23-33}$.

Epidemiological studies are necessary for the implementation of effective prevention measures. Genotyping of strains from patients in odontological clinical treatment and their environments can provide information that can potentially help control and prevent the spread of $S$. aureus involved in the processes of colonization and human infection. This scientific research evaluated the genetic diversity of natural populations of oxacillin-resistance $S$. aureus dental isolates (dental patients and environments). The 
frequency of strains and operational taxonomic groups (taxon and cluster) and possible epidemiological correlations were investigated by using isoenzymatic markers (MLEE) and genetic and grouping analysis.

\section{Material and Methods}

\section{Microbiological Sampling}

A total of ninety-nine bacterial samples of oxacillin-resistant S. aureus (ORSA), from the bacteria collection of the Laboratório de Farmacogenética e Biologia Molecular, Faculdade de Ciências Médicas and Centro de Pesquisa e Pós-graduação (UNIFENAS), Alfenas, MG, Brazil, were kindly provided and used for the present research. These samples were previously isolated from dental patients and clinical environment (air) (Faculdade de Odontologia, UNIFENAS) (approved by Committee of Ethics in Human Research, protocol no. 174/2009) and characterized using microbiological methods of identification [i.e., stain of Gram, growth in chromogenic medium CHROMagar Staphylococcus aureus ${ }^{\circledR}$, catalase test, coagulase test (Coagu-Plasma, Laborclin Produtos para Laboratórios Ltda.), clumping factor A test (Staphy Test, Probac do Brasil Produtos Bacteriológicos Ltda.), fermentation of mannitol test and DNAse test ${ }^{34}$ and antimicrobial susceptibility testing (i.e., diffusion disk and confirmatory triage for resistance to oxacillin) ${ }^{35}$.

\section{Multilocus Enzyme Electrophoresis (MLEE)}

Preparation of cell extracts, electrophoresis procedures, enzyme staining and genetic interpretation of MLEE patterns were performed according to methods previously reported $^{23,25,26,31}$. To ensure reproducibility of the results, the cellular enzymes of the $S$. aureus ATCC ${ }^{\circledR} 25.923^{\mathrm{TM}}$ reference strain were systematically used. A total of nine metabolic enzymes (Table 1) was investigated using systems and solutions previously established for the MLEE analyses $23,25,26,31$. The discriminatory power of the MLEE method was determined using the numeric index of discrimination $(D)$, in accordance with the probability that two unrelated isolates sampled from a test population are classified into different types (i.e., strains or ETs) ${ }^{25,26}$.

\section{Grouping Analysis}

The statistic of Nei $(1972)^{36}$ was used to estimate the genetic distance $\left(d_{\mathrm{ij}}\right)$ among the isolates/strains (ETs) of oxacillin-resistant $S$. aureus. The interpretation in terms of enzyme loci infers that, on average, from zero to an infinite number of allele substitutions are detected (for electrophoresis) for every 100 existing loci from a common ancestral strain. A tree with two-dimensional classification (dendrogram), based on the matrix $d_{i j}$, was generated by the grouping SAHN method (Sequential, Agglomerative, Hierarchic, Nonoverlapping Clustering Methods) and the UPGMA algorithm (Unweighted Pair-Group Method Using an Arithmetic Average). Once MLEE provided all levels of relationship that must be solved by DNA fingerprinting methods (i.e., identification of the same strain between independent isolates, identification of microevolutionary changes in the same strain, identification of clusters of moderately related isolates and identification of completely unrelated isolates), a threshold (average value: $\overline{d_{i j}}$ ) in the dendrogram was established to identify identical isolates and highly related isolates, clusters 
Table 1. Systems and solutions utilized for the MLEE analyses of the S. aureus metabolic enzymes.

\begin{tabular}{|c|c|c|c|c|c|c|c|}
\hline \multicolumn{3}{|c|}{ Enzyme } & \multicolumn{5}{|c|}{ Compound for staining } \\
\hline $\begin{array}{l}\text { EC } \\
\text { number }\end{array}$ & Name & Symbol & Substrate & Buffer & Salt & Coenzyme & $\begin{array}{l}\text { Dye and } \\
\text { Catalyser }\end{array}$ \\
\hline 1.1.1.1. & $\begin{array}{c}\text { alcohol } \\
\text { dehydrogenase }\end{array}$ & $\mathrm{ADH}$ & $\begin{array}{c}\text { Ethanol (3mL) } \\
\text { Isopropanol } \\
(2 \mathrm{~mL})\end{array}$ & $\begin{array}{c}200 \mathrm{mM} \\
\text { Tris- } \mathrm{HCl} \mathrm{pH} \\
8.0 \text { (q.s.p. } \\
50 \mathrm{~mL} \text { ) }\end{array}$ & & $\begin{array}{l}\text { NAD 1\% } \\
(2 \mathrm{~mL})\end{array}$ & $\begin{array}{c}\text { PMS 1\% } \\
(500 \mu \mathrm{L}) \mathrm{MTT} \\
1.25 \%(1 \mathrm{~mL})\end{array}$ \\
\hline
\end{tabular}

\begin{tabular}{|c|c|c|c|c|c|c|}
\hline 1.1 .1 .17 & $\begin{array}{c}\text { mannitol-1- } \\
\text { phosphate } \\
\text { dehydrogenase }\end{array}$ & M1P & $\begin{array}{c}\text { Mannitol } \\
\text { 1-phosphate } \\
(5 \mathrm{mg})\end{array}$ & $\begin{array}{c}200 \mathrm{mM} \\
\text { Tris-HCl pH } \\
8.0 \text { (q.s.p. } \\
50 \mathrm{~mL} \text { ) }\end{array}$ & $\begin{array}{c}\text { NAD 1\% } \\
(2 \mathrm{~mL})\end{array}$ & $\begin{array}{c}\text { PMS 1\% } \\
(500 \mu \mathrm{L}) \mathrm{MTT} \\
1.25 \%(1 \mathrm{~mL})\end{array}$ \\
\hline
\end{tabular}

\begin{tabular}{|c|c|c|c|c|c|c|}
\hline 1.1.1.37. & $\begin{array}{c}\text { malate } \\
\text { dehydrogenase }\end{array}$ & $\mathrm{MDH}$ & $\begin{array}{l}\text { 2M Malic acid } \\
\quad(6 \mathrm{~mL})^{b}\end{array}$ & $\begin{array}{c}200 \mathrm{mM} \\
\text { Tris-HCl pH } \\
8.0 \text { (q.s.p. } \\
50 \mathrm{~mL})^{\mathrm{a}}\end{array}$ & $\begin{array}{l}\text { NAD 1\% } \\
(2 \mathrm{~mL})\end{array}$ & $\begin{array}{c}\text { PMS 1\% } \\
(500 \mu \mathrm{L}) \mathrm{MTT} \\
1.25 \%(1 \mathrm{~mL})\end{array}$ \\
\hline
\end{tabular}

\begin{tabular}{|c|c|c|c|c|c|c|c|}
\hline 1.1.1.47 & $\begin{array}{c}\text { glucose } \\
\text { dehydrogenase }\end{array}$ & GDH & $\begin{array}{l}\text { D-glucose } \\
(500 \mathrm{mg})\end{array}$ & $\begin{array}{c}200 \mathrm{mM} \\
\text { Tris-HCl pH } \\
8.0 \text { (q.s.p. } \\
50 \mathrm{~mL})^{\mathrm{a}}\end{array}$ & & $\begin{array}{l}\text { NAD 1\% } \\
(2 \mathrm{~mL})\end{array}$ & $\begin{array}{c}\text { PMS 1\% } \\
(500 \mu \mathrm{L}) \mathrm{MTT} \\
1.25 \%(1 \mathrm{~mL})\end{array}$ \\
\hline 1.1.1.48 & $\begin{array}{c}\text { D-galactose } \\
\text { dehydrogenase }\end{array}$ & GLDH & $\begin{array}{l}\text { Galactose } \\
(450 \mathrm{~mL})\end{array}$ & $\begin{array}{c}\text { Tris-HCl } \\
100 \mathrm{mM} \mathrm{pH} \\
8.4 \text { (q.s.p. } \\
50 \mathrm{~mL})^{\circ}\end{array}$ & & $\begin{array}{l}\text { NAD 1\% } \\
(1 \mathrm{~mL})\end{array}$ & $\begin{array}{c}\text { PMS 1\% } \\
(500 \mu \mathrm{L}) \mathrm{MTT} \\
1.25 \%(1 \mathrm{~mL})\end{array}$ \\
\hline 1.1.1.49 & $\begin{array}{c}\text { glucose-6- } \\
\text { phosphate } \\
\text { dehydrogenase }\end{array}$ & G6PDH & $\begin{array}{l}\text { Glicose-6- } \\
\text { phosphate } \\
\text { disodium salt } \\
(100 \mathrm{mg})\end{array}$ & $\begin{array}{c}200 \mathrm{mM} \\
\text { Tris- } \mathrm{HCl} \mathrm{pH} \\
8.0 \text { (q.s.p. } \\
50 \mathrm{~mL} \text { ) }{ }^{a}\end{array}$ & $\begin{array}{l}100 \mathrm{mM} \\
\mathrm{MgCl}_{2} \\
(1 \mathrm{~mL})^{\mathrm{d}}\end{array}$ & $\begin{array}{l}\text { NADP } 1 \% \\
(1 \mathrm{~mL})\end{array}$ & $\begin{array}{c}\text { PMS 1\% } \\
(500 \mu \mathrm{L}) \mathrm{MTT} \\
1.25 \%(1 \mathrm{~mL})\end{array}$ \\
\hline
\end{tabular}

1.11.1.6 catalase CAT $^{\mathrm{e}}$

\begin{tabular}{|c|c|c|c|c|c|}
\hline 3.1.1.1. & $\begin{array}{l}\alpha \text { - and } \beta \text { - } \\
\text { esterase }\end{array}$ & EST & $\begin{array}{c}\alpha-\text { and } \\
\beta-\text { Naphthyl } \\
\text { acetate }(1 \% \\
\text { solution in } \\
\text { acetone }) \\
(1.5 \mathrm{ml})\end{array}$ & $\begin{array}{c}50 \mathrm{mM} \\
\text { Sodium } \\
\text { phosphate } \\
\text { pH } 7.0 \\
\text { (q.s.p. } 50 \mathrm{~mL})^{\mathrm{f}}\end{array}$ & $\begin{array}{l}\text { Fast Blue RR } \\
\text { salt }(25 \mathrm{mg})\end{array}$ \\
\hline
\end{tabular}

Electrode buffer: Tris-citrate $\mathrm{pH} 8.0$ [83.2 $\mathrm{g}$ of $\mathrm{C}_{4} \mathrm{H}_{11} \mathrm{NO}_{3}$ (Tris), $33.09 \mathrm{~g}$ of $\mathrm{C}_{6} \mathrm{H}_{8} \mathrm{O}_{7} \cdot \mathrm{H}_{2} \mathrm{O}$ (Citric acid), 1 liter of $\mathrm{H}_{2} \mathrm{O}$; Gel buffer: Electrode buffer diluted 1:29.

a $24.2 \mathrm{~g}$ of $\mathrm{C}_{4} \mathrm{H}_{11} \mathrm{NO}_{3}$ (Tris), 1 liter of $\mathrm{H}_{2} \mathrm{O}$ ( $\mathrm{pH}$ adjusted with $\mathrm{HCl}$ );

b $26.8 \mathrm{~g}$ of $\mathrm{C}_{4} \mathrm{H}_{6} \mathrm{O}_{5}$ (DL-malic acid) and $16 \mathrm{~g}$ of $\mathrm{NaOH}$ in $100 \mathrm{ml}$ of $\mathrm{H}_{2} \mathrm{O}$ (caution: potentially explosive reaction);

c $12.1 \mathrm{~g}$ of $\mathrm{C}_{4} \mathrm{H}_{11} \mathrm{NO}_{3}$ (Tris), 1 liter of $\mathrm{H}_{2} \mathrm{O}$ (pH adjusted with $\mathrm{HCl}$ );

d $2.03 \mathrm{~g}$ of $\mathrm{MgCl}_{2} \cdot 6 \mathrm{HCl}$ (Magnesium chloride) in $100 \mathrm{ml}$ of $\mathrm{H}_{2} \mathrm{O}$;

e Incubate gel slice for $30 \mathrm{~min}$ at $0{ }^{\circ} \mathrm{C}$ in $50 \mathrm{ml}$ of $0.1 \mathrm{M}$ sodium phosphate $\mathrm{pH} 7.0$ buffer, then pour off solution, and immerse it in $50 \mathrm{ml}$ of $1.5 \%$ potassium iodide solution $(\mathrm{KI})$ for $2 \mathrm{~min}$. Therefore, rinse gel slice with water, and immerse it in $50 \mathrm{ml}$ of $0.03 \%$ hydrogen peroxide $\left(\mathrm{H}_{2} \mathrm{O}_{2}\right)$ solution. Mix gently and remove stain solution when white zones appear on dark-blue background;

f Sodium phosphate buffer $\mathrm{pH}$ 7.0: mix equal parts of $27.6 \mathrm{~g}$ of $\mathrm{NaH}_{2} \mathrm{PO}_{4} \cdot \mathrm{H}_{2} \mathrm{O}$ (monobasic) in 1 liter of water and $53.6 \mathrm{~g}$ of $\mathrm{Na}_{2} \mathrm{HPO}_{4} \cdot 7 \mathrm{H}_{2} \mathrm{O}$ in 1 liter of water, then dilute the mixture $1: 25$ with water. 
$\left(0 \leq d_{i j}<\overline{d_{i j}}\right)$ and taxa (singular taxon, i.e., taxonomic group of any nature or rank) $\left(d_{i j} \geq \overline{d_{i j}}\right)$. Correlation coefficients based on the Pearson product-moment was used as a measure of agreement between the genetic distance values implicit in the UPGMA dendrogram and the original explicit values in the matrix of genetic distance $d_{i j}$. All these analyses were performed using the NTSYS-pc program version 2.125,26,32.

\section{Results}

The electrophoretic isoenzyme patterns of oxacillin-resistant $S$. aureus isolates were reproducible in different gels after three repetitions of each electrophoretic run. According to haploid nature of $S$. aureus, these patterns displayed the following characteristics (Table 2): all 30 enzymatic loci were polymorphic for one, two, three, four, five and six alleles (one allele: Cat-2, $\beta$-Est-3, Gdh-2, G6pdh-3, Sdh-1; two alleles: Adh-1, Cat-1, $\alpha$-Est-1, $\beta$-Est-2, M1p-2, M1p-3, Mdh-3 and Sdh-2; three alleles: Gdh-3, Mdh-1, Sdh-3 and Mdh-4; four alleles: Adh-2, Adh-3, $\alpha$-Est-3, $\beta$-Est-1, G6pdh-2 and Mdh-2; five alleles: M1p-1, Gdh-1, Gldh-2 and G6pdh-4; six alleles: $\alpha$-Est-2, G6pdh-1 and Gldh-1). The average number of alleles per polymorphic locus was equal to $3.16 \pm 1.62$. The existing combination in 30 enzymatic loci revealed 79 strains (ETs) [79\% of the isolates, including the reference strain of S. aureus ATCC $^{\circledR} 25.923$, that is, identical isolates that match the same strain $\left.{ }^{\mathrm{ET}}\left(d_{\mathrm{ij}}=0.000\right)\right]$. Based on the genetic interpretation of electrophoretic patterns, the discriminatory power of the MLEE genotyping method was equal to 0.99051, that is, there was a $99 \%$ probability that two non-related isolates of $S$. aureus, from the test population, would be classified as distinct strains ${ }^{\mathrm{ET}}$.

The genetic diversity among the strains of oxacillin-resistant $S$. aureus was evaluated using the matrix $d_{\mathrm{ij}}$ and the UPGMA dendrogram (Figure 1). Considering the threshold obtained $\left(0 \leq d_{i j}<0.282\right.$ : isolates identical or highly related; $0.282 \leq d_{i j}<0.653$ : isolates moderately related; $d_{i j} \geq 0.653$ : isolates genetically distantly related), the results indicated three main groups or taxa, designated A, B and C. Taxon A comprised nine isolates/strains (ET2 G22.5, ET6 G11.66, ET8 G11.86, ET9 G11.129, ET22 G18.66, ET24 G18.8, ET32 G18.46, ET68 ${ }^{\mathrm{G} 11.36}$ and ET76 ${ }^{\mathrm{G18.137}}$ ) and eight moderately related clusters (from I to VIII; a total of 60 isolates ${ }^{60 \%}$ or 43 ETs ${ }^{54.4 \%}$ ):

- Cluster I: Thirteen identical and/or highly related isolates, including the reference strain; 11 highly related strains (ET1 ATCC 25.923 and G13.172, ET41 G18.100 and G20.44, ET43 G20.12, ET44 G18.155, ET45 G22.55, ET46 622.22, ET47 G18.51, ET48 G15.100, ET49 G16.140, ET50 G13.165 and ET5 $1^{\mathrm{G} 15.40}$ ).

- Cluster II: Five identical and/or highly related isolates; two highly related strains (ET36 ${ }^{\mathrm{G} 18.20}$ and ET39 G18.110, G18.111, G18.166 and G18.156).

- Cluster III: Three highly related isolates; two highly related strains (ET13 G11.135, ET15 ${ }^{\mathrm{G} 13.47}$ and ET2 ${ }^{\mathrm{G} 18.95}$ ).

- Cluster IV: Three highly related isolates; three highly related strains (ET3 66.15, ET5 G5.38 and ET4 ${ }^{\mathrm{G} 6.12}$ ).

- Cluster V: Four identical and/or highly related isolates; three highly related strains

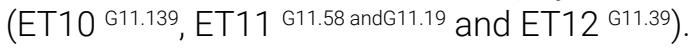


Table 2. Allele profiles of oxacillin-resistant $S$. aureus isolates (79 strains/ETs), sourced from odontological clinical and environment samples, and obtained for the genetic interpretation of MLEE patterns. Discriminatory power $(D)=0.99051$. (-) allele null.

\begin{tabular}{|c|c|c|c|c|c|c|c|c|c|c|c|c|c|c|c|c|c|c|c|c|c|c|c|c|c|c|c|c|c|c|}
\hline \multirow{3}{*}{ ET } & \multicolumn{30}{|c|}{ Alleles in 30 enzyme loci } \\
\hline & \multicolumn{3}{|c|}{ Adh } & \multicolumn{3}{|c|}{$S d h$} & \multicolumn{3}{|c|}{ M1p } & \multicolumn{4}{|c|}{ Mdh } & \multicolumn{3}{|c|}{ Gdh } & \multicolumn{2}{|c|}{ Gldh } & \multicolumn{4}{|c|}{ G6pdh } & \multicolumn{2}{|c|}{ Cat } & \multicolumn{3}{|c|}{$\mathrm{a}-E s t$} & \multicolumn{3}{|c|}{$\beta$-Est } \\
\hline & 1 & 2 & 3 & 1 & 2 & 3 & 1 & 2 & 3 & 1 & 2 & 3 & 4 & 1 & 2 & 3 & 1 & 2 & 1 & 2 & 3 & 4 & 1 & 2 & 1 & 2 & 3 & 1 & 2 & 3 \\
\hline ET1 & - & - & 2 & - & 2 & - & - & 2 & & 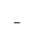 & - & 1 & - & - & 1 & 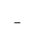 & - & 1 & 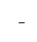 & - & 1 & & 2 & - & - & 5 & 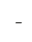 & - & 1 & - \\
\hline ET2 & - & - & 2 & - & 2 & - & - & 2 & & 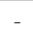 & 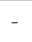 & 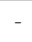 & 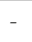 & - & 1 & - & 6 & 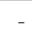 & 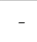 & - & 1 & & 2 & - & - & 5 & 2 & - & - & - \\
\hline ET3 & - & - & 2 & - & 2 & 1 & - & - & 1 & - & 2 & - & - & - & 1 & 2 & - & 3 & - & 2 & - & - & 2 & - & - & 5 & - & - & 1 & - \\
\hline ET4 & 2 & - & - & - & 2 & 1 & - & - & 1 & - & 2 & - & - & - & 1 & 2 & - & 3 & - & 2 & - & & 2 & - & - & 6 & 4 & - & 2 & - \\
\hline ET5 & - & - & 2 & - & - & 1 & - & - & 1 & - & 4 & - & - & - & 1 & 2 & - & 3 & - & 2 & - & - & 2 & - & - & 5 & - & - & 1 & - \\
\hline ET6 & - & 2 & 1 & & 2 & 1 & - & 2 & 1 & - & - & 1 & 2 & - & - & - & 6 & 2 & - & 4 & - & - & 2 & - & - & & - & - & - & \\
\hline ET7 & - & - & - & - & 2 & 1 & - & 2 & 1 & 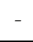 & - & - & - & - & - & - & - & - & - & 4 & - & 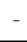 & 2 & - & - & - & - & 4 & - & - \\
\hline ET8 & - & - & - & - & 2 & 1 & - & 2 & 1 & - & - & - & 1 & - & - & - & - & - & - & - & 1 & - & 2 & - & - & 1 & - & - & - & - \\
\hline ET9 & - & - & - & - & 2 & 1 & - & 2 & 1 & - & - & - & - & - & - & - & - & 4 & - & - & 1 & - & 2 & - & - & - & 1 & - & - & - \\
\hline ET10 & - & 4 & 2 & - & 2 & - & 5 & 2 & - & - & 4 & 1 & - & 5 & 1 & - & 5 & 1 & - & - & 1 & 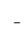 & 2 & - & - & 5 & - & - & 1 & - \\
\hline ET11 & - & 4 & - & - & 2 & - & 4 & - & - & - & 4 & - & - & 5 & - & - & 5 & - & - & - & - & 3 & 2 & - & - & 3 & - & - & 1 & - \\
\hline ET12 & - & 4 & - & - & 2 & - & 4 & - & - & - & 4 & - & - & 3 & - & - & 5 & - & - & - & 1 & 3 & 2 & - & - & 3 & - & - & 1 & - \\
\hline ET13 & - & 1 & - & - & - & - & 2 & - & - & 3 & - & - & - & - & - & - & 2 & - & - & - & - & 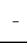 & 2 & - & - & 5 & - & - & - & - \\
\hline ET14 & - & - & - & - & - & - & - & - & - & - & 3 & - & - & 5 & - & - & - & - & - & - & 1 & 4 & 2 & - & - & 5 & - & - & - & - \\
\hline ET15 & - & - & - & - & - & - & - & - & 1 & 3 & - & - & - & - & - & - & - & - & - & 2 & - & - & 2 & - & - & 5 & - & - & 1 & 1 \\
\hline ET16 & - & - & - & - & - & - & - & - & 1 & 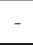 & - & - & - & 5 & - & - & 3 & - & - & - & 1 & & 2 & - & - & 2 & - & - & - & - \\
\hline ET17 & - & - & - & - & 2 & - & - & - & 7 & - & - & - & - & - & - & - & - & - & - & 4 & - & - & 2 & - & - & - & - & - & - & - \\
\hline ET18 & - & - & - & & - & - & - & & 1 & - & - & - & & - & - & - & & & 4 & - & & & 2 & - & - & 2 & - & - & - & \\
\hline ET19 & - & - & 2 & & - & 3 & - & 1 & & 3 & - & - & & - & - & - & & - & 6 & - & & & 2 & - & - & & - & - & - & \\
\hline ET20 & - & 2 & - & - & 2 & - & - & - & 1 & 3 & - & 2 & - & 5 & - & - & - & - & - & - & 1 & - & 2 & - & - & - & - & - & - & - \\
\hline ET21 & - & - & 2 & - & - & 3 & - & 1 & - & 3 & - & - & - & - & - & - & - & 1 & - & - & - & - & 2 & - & - & 5 & - & - & - & - \\
\hline ET22 & - & - & - & - & - & - & 3 & - & 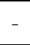 & - & 3 & - & - & - & - & - & 4 & - & - & - & 1 & 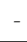 & 2 & - & - & 5 & - & - & - & - \\
\hline ET23 & - & - & - & - & - & - & - & - & 1 & - & 3 & - & - & - & - & - & - & - & - & 2 & - & - & 2 & - & - & - & 1 & 2 & - & - \\
\hline ET24 & - & - & - & - & - & - & - & - & 1 & - & 3 & - & 2 & - & - & - & - & - & - & 2 & - & - & 2 & - & - & 5 & - & - & - & 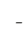 \\
\hline ET25 & - & - & 2 & 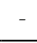 & - & 3 & - & - & 1 & - & 4 & - & - & 5 & - & - & - & - & 6 & - & - & 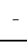 & 2 & - & - & - & - & - & 1 & 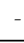 \\
\hline ET26 & - & - & - & - & - & - & - & - & 1 & - & 4 & - & - & - & - & - & - & 1 & - & - & 1 & - & 2 & - & - & 2 & - & 2 & - & - \\
\hline ET27 & - & - & 2 & - & 2 & - & - & 2 & - & - & - & - & 3 & - & 1 & - & - & 1 & - & - & 1 & - & 2 & - & 1 & - & - & - & 1 & - \\
\hline ET28 & - & - & 2 & - & 2 & - & 5 & 2 & - & - & - & - & 3 & - & 1 & - & - & 1 & - & - & 1 & - & 2 & - & 1 & - & - & - & 1 & - \\
\hline ET29 & - & - & 2 & - & 2 & - & - & 2 & - & - & - & 1 & 3 & - & 1 & - & - & 1 & - & - & 1 & - & 2 & - & 2 & - & - & - & 1 & - \\
\hline ET30 & - & - & 2 & - & 2 & - & - & 2 & - & - & - & 1 & - & - & - & - & - & 1 & - & - & 1 & - & 2 & - & 2 & - & - & - & 1 & - \\
\hline ET31 & - & - & 2 & - & 2 & - & - & 2 & 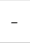 & - & - & - & - & 1 & - & - & - & 1 & - & - & 1 & - & 2 & - & 2 & - & - & - & 1 & . \\
\hline ET32 & - & 3 & - & - & 2 & - & - & 2 & - & - & - & 1 & - & 2 & - & - & - & 1 & - & - & 1 & - & 2 & - & 2 & - & - & - & 1 & - \\
\hline ET33 & - & - & 2 & - & 2 & - & - & 2 & - & - & - & 1 & - & - & 1 & - & - & 1 & - & - & 1 & - & 2 & - & 2 & - & - & - & 1 & - \\
\hline ET34 & - & - & 2 & - & 2 & - & - & 2 & 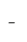 & - & - & 1 & - & - & 1 & - & - & 1 & - & - & 1 & - & 2 & - & - & - & - & - & 1 & - \\
\hline ET35 & - & - & 2 & - & 2 & - & - & 2 & - & - & - & 1 & - & 3 & 1 & - & - & 1 & - & - & 1 & - & 2 & - & - & - & - & 3 & - & - \\
\hline ET36 & - & - & 2 & - & 2 & - & 5 & 2 & 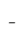 & - & - & 1 & - & - & 1 & - & - & 1 & - & - & 1 & 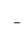 & 2 & - & 2 & 6 & - & 3 & - & - \\
\hline ET37 & - & - & 2 & - & 2 & - & - & 2 & - & - & - & 1 & - & 3 & - & - & - & 1 & - & - & 1 & - & 2 & - & 2 & - & - & 3 & - & - \\
\hline ET38 & - & - & 2 & - & 2 & - & 5 & 2 & - & - & - & 1 & - & - & 1 & - & - & 1 & - & - & 1 & - & 2 & - & 2 & - & - & 3 & - & - \\
\hline ET39 & - & - & 2 & - & 2 & - & 5 & 2 & 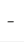 & - & 3 & - & - & - & 1 & - & - & 1 & - & - & 1 & & 2 & - & - & 6 & - & 3 & - & . \\
\hline ET40 & - & - & 2 & - & 2 & - & 5 & 2 & - & - & - & 1 & - & - & 1 & - & - & 1 & - & - & 1 & - & 2 & - & 1 & - & - & 3 & - & - \\
\hline ET41 & - & - & 2 & - & 2 & - & - & 2 & - & - & - & 1 & & - & 1 & - & - & 1 & - & - & 1 & - & 2 & - & - & 6 & - & 3 & - & - \\
\hline
\end{tabular}

ET and [-] correspond to electrophoretic type (bacterial strain) and allele null, respectively. Continue 
Table 2. Allele profiles of oxacillin-resistant $S$. aureus isolates (79 strains/ETs), sourced from odontological clinical and environment samples, and obtained for the genetic interpretation of MLEE patterns. Discriminatory power $(D)=0.99051$. (-) allele null. Continuation

\begin{tabular}{|c|c|c|c|c|c|c|c|c|c|c|c|c|c|c|c|c|c|c|c|c|c|c|c|c|c|c|c|c|c|c|c|}
\hline \multirow{3}{*}{ ET } & \multicolumn{31}{|c|}{ Alleles in 30 enzyme loci } \\
\hline & \multicolumn{3}{|c|}{ Adh } & \multicolumn{3}{|c|}{$S d h$} & \multicolumn{3}{|c|}{ M1p } & \multicolumn{4}{|c|}{ Mdh } & \multicolumn{3}{|c|}{ Gdh } & \multicolumn{2}{|c|}{ Gldh } & \multicolumn{4}{|c|}{ G6pdh } & \multicolumn{2}{|c|}{ Cat } & \multicolumn{4}{|c|}{$\mathrm{a}-E s t$} & \multicolumn{3}{|c|}{$\beta$-Est } \\
\hline & 1 & 2 & 3 & 1 & 2 & 3 & 1 & 2 & 3 & 1 & 2 & 3 & 4 & 1 & 2 & 3 & 1 & 2 & 1 & 2 & 3 & 4 & 1 & 2 & 1 & & 2 & 3 & 1 & & 3 \\
\hline ET42 & - & - & 2 & - & 2 & - & - & 2 & - & - & - & - & & - & 1 & - & - & & - & - & 1 & & 2 & - & 1 & 1 & - & - & 3 & & - \\
\hline ET43 & - & - & 2 & - & 2 & - & - & & & - & 3 & - & & - & 1 & - & - & & - & - & 1 & & 2 & - & - & & 5 & - & - & & - \\
\hline ET44 & - & - & 2 & - & 2 & - & - & 2 & 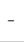 & - & 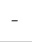 & - & & - & 1 & - & - & & 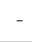 & 3 & - & & 2 & - & - & - & 5 & - & - & & 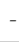 \\
\hline ET45 & - & - & 2 & - & 2 & - & - & 2 & - & - & - & - & & - & 1 & - & - & & - & - & 1 & . & 2 & - & - & - & 5 & - & - & 1 & - \\
\hline ET46 & - & - & 2 & & 2 & - & - & 2 & & - & - & 1 & & & 1 & - & & 1 & - & - & 1 & & 2 & - & & & 5 & & 1 & - & - \\
\hline ET47 & - & - & 2 & - & 2 & - & - & 2 & - & - & - & - & 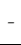 & - & 1 & - & - & 1 & - & 3 & 1 & 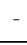 & 2 & - & - & - & 5 & - & - & I & - \\
\hline ET48 & - & - & 2 & - & 2 & - & - & 2 & - & - & - & - & & - & 1 & - & - & 7 & - & - & 1 & . & 2 & - & - & - & 5 & - & 3 & 1 & \\
\hline ET49 & - & - & 2 & - & 2 & - & - & 2 & - & 2 & - & - & & - & 1 & - & - & 1 & - & - & 1 & & 2 & - & - & - & 4 & - & 3 & & - \\
\hline ET50 & - & - & 2 & - & 2 & - & - & 2 & - & 2 & - & - & - & - & 1 & - & - & 1 & - & - & 1 & 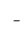 & 2 & - & & - & 5 & - & - & 1 & - \\
\hline ET51 & - & - & 2 & - & 2 & - & - & 2 & - & 2 & - & - & & - & 1 & - & - & 7 & - & - & 1 & - & 2 & - & - & - & 4 & - & - & 7 & - \\
\hline ET52 & - & - & - & - & - & - & - & - & - & 2 & - & - & 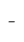 & - & - & - & - & 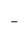 & - & 1 & 1 & . & 2 & - & - & - & - & - & - & - & - \\
\hline ET53 & - & - & 4 & - & - & 2 & 3 & - & 2 & 2 & - & - & 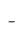 & - & - & 3 & 1 & 5 & 5 & - & - & 5 & 2 & 1 & - & 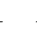 & - & - & - & 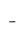 & - \\
\hline ET54 & & - & - & - & & - & - & - & & 2 & 1 & - & & & - & - & - & & & 1 & - & 2 & 2 & - & & & - & - & & 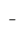 & - \\
\hline ET55 & - & - & - & - & - & - & - & - & - & 2 & - & - & - & - & - & - & - & - & 3 & - & - & - & 2 & - & - & 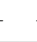 & - & - & - & 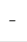 & - \\
\hline ET56 & - & - & - & - & - & - & - & - & - & - & 1 & - & 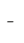 & - & - & - & - & 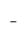 & 1 & - & - & 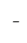 & 2 & - & - & - & - & 2 & - & 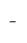 & - \\
\hline ET57 & 1 & 3 & - & - & - & - & - & - & - & - & 1 & - & - & - & - & - & - & - & - & - & - & 2 & 2 & - & - & - & - & - & - & 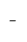 & - \\
\hline ET58 & - & - & - & - & - & - & - & - & - & - & - & - & 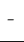 & - & - & - & - & - & 1 & - & - & - & 2 & - & - & - & - & - & - & 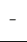 & - \\
\hline ET59 & - & - & & & - & - & - & - & & - & 1 & - & & - & - & - & & & 3 & - & - & & 2 & - & - & - & & - & - & 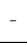 & - \\
\hline ET60 & - & - & 3 & 1 & - & - & - & - & - & - & 1 & - & - & 5 & - & - & - & - & - & - & - & 5 & 2 & - & - & - & - & - & - & - & - \\
\hline ET61 & - & - & - & 1 & 1 & - & - & - & - & 2 & - & - & - & 4 & - & 1 & - & - & 1 & - & - & - & 2 & - & & - & - & - & - & 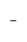 & - \\
\hline ET62 & - & - & - & - & - & - & - & - & - & - & - & - & 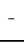 & - & - & - & - & 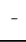 & - & - & - & - & 2 & - & - & - & - & - & - & 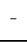 & - \\
\hline ET63 & 1 & 3 & - & - & - & - & - & - & - & - & - & - & - & - & - & - & - & - & 2 & 1 & - & - & 2 & - & - & - & - & - & 4 & - & - \\
\hline ET64 & - & - & - & - & - & - & - & - & - & - & - & - & 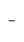 & - & - & - & - & 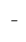 & 2 & - & - & - & 2 & - & & - & - & - & - & - & - \\
\hline ET65 & - & - & - & - & - & - & - & - & - & 1 & - & - & - & - & - & - & - & - & 5 & - & - & - & 2 & - & & - & - & - & - & 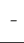 & - \\
\hline ET66 & - & - & - & - & - & - & - & - & - & - & - & - & - & 4 & - & - & - & - & - & - & 1 & - & 2 & - & - & - & - & - & - & - & - \\
\hline ET67 & - & - & - & - & - & - & - & - & - & - & - & - & - & - & - & - & - & 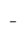 & - & - & - & - & 2 & - & 1 & 7 & - & - & - & 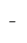 & - \\
\hline ET68 & - & - & - & - & - & - & 4 & - & - & - & - & - & - & - & - & - & 5 & - & - & 1 & - & - & 2 & - & 2 & 2 & - & - & - & - & - \\
\hline ET69 & - & - & - & - & - & - & - & - & - & 2 & - & - & - & - & - & - & - & - & - & - & - & - & 2 & - & - & - & - & - & - & - & - \\
\hline ET70 & - & - & - & - & - & - & - & - & - & 1 & - & - & - & 4 & - & - & - & 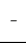 & - & - & - & - & 2 & - & - & - & - & - & - & 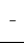 & - \\
\hline ET71 & - & - & - & - & - & - & - & - & - & 1 & - & - & - & - & - & - & - & 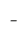 & 2 & 1 & - & - & 2 & - & - & - & - & - & - & - & - \\
\hline ET72 & - & - & 4 & - & - & - & - & - & - & - & - & - & - & - & - & - & & & 5 & - & - & & 2 & - & & & & & - & - & - \\
\hline ET73 & - & - & - & - & - & - & - & - & - & - & - & - & - & - & - & - & - & 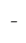 & 2 & 1 & - & - & 2 & - & - & - & - & - & - & - & - \\
\hline ET74 & - & - & - & - & - & - & 1 & - & - & - & - & 1 & . & - & - & - & 1 & 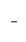 & 5 & - & - & - & 2 & - & - & - & - & - & - & - & - \\
\hline ET75 & - & - & - & - & - & - & - & - & - & - & - & 1 & & - & 1 & - & - & & - & 3 & - & - & 1 & - & - & - & - & - & - & - & - \\
\hline ET76 & - & - & - & - & - & - & - & - & - & - & - & 1 & 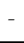 & - & 1 & - & - & 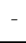 & - & 3 & - & - & 1 & - & - & - & 5 & 3 & - & - & - \\
\hline ET77 & - & - & - & - & - & - & - & - & - & - & - & 2 & 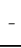 & - & - & & & - & - & 2 & & 1 & 1 & - & & & & - & - & 1 & \\
\hline ET78 & - & - & - & - & - & - & - & - & - & - & - & 2 & & - & - & - & - & - & - & 3 & - & - & 1 & - & - & - & - & - & - & - & - \\
\hline ET79 & - & - & - & - & - & - & - & 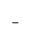 & & 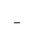 & - & $z$ & & 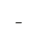 & - & - & - & & 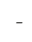 & - & 1 & - & 1 & - & - & & - & - & - & - & \\
\hline
\end{tabular}

ET and [-] correspond to electrophoretic type (bacterial strain) and allele null, respectively. 


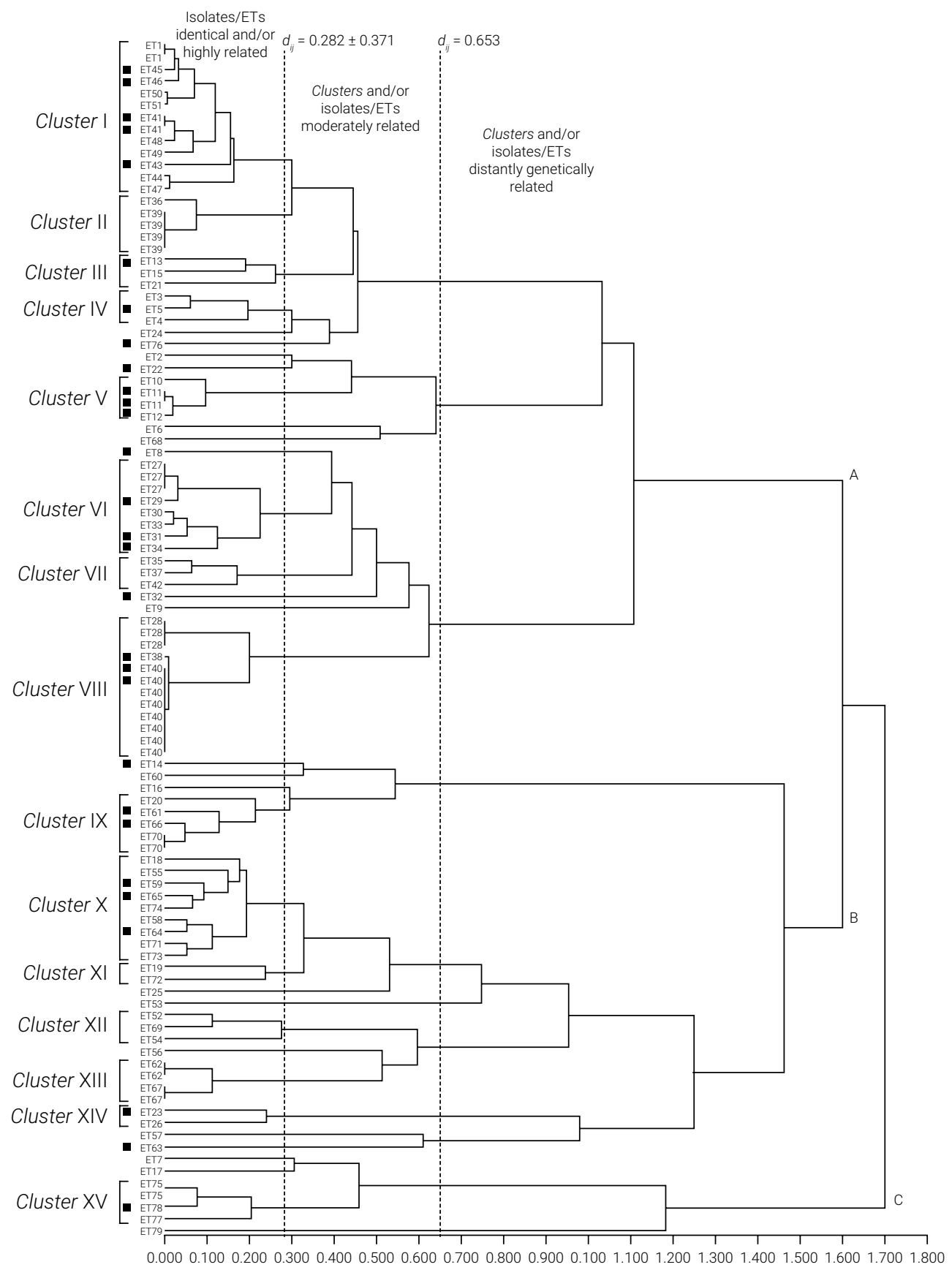

Figure 1. Genetic diversity of 99 oxacillin-resistant $S$. aureus isolates sourced from a population of odontological clinical and environment samples. The UPGMA dendrogram $\left(r_{j k}=0.79908\right)$ was generated from a matrix of genetic distance $d_{i j}(\mathrm{Nei}, 1972)$.

- Cluster VI: Eight identical and/or highly related isolates; six highly related strains (ET27 618.33, G18.104 and G20.45, ET29 ${ }^{\text {G18.14, ET30 }}{ }^{\text {619.43 }}$, ET31 ${ }^{\mathrm{G} 22.48}$, ET33 ${ }^{\mathrm{G} 18.135}$ and ET34 ${ }^{\mathrm{G} 20.14}$ ).

- Cluster VII: Three highly related isolates; three highly related strains (ET35 G18.45, ET37 ${ }^{\text {G19.10 }}$ and ET42 ${ }^{\text {G18.126). }}$. 
- Cluster VIII: Twelve identical and/or highly related isolates; three highly related strains (ET28 G18.94, G18.9 and G21.1, ET38 G18.55, ET40 G15.52, G16.40, G18.142, G18.26, G18.50, G18.74, G22.63 and G19.21).

Taxon B comprised eight isolates/strains (ET14 G13.112, ET60 G14.71, ET16 G12.14, ET25 G18.89,

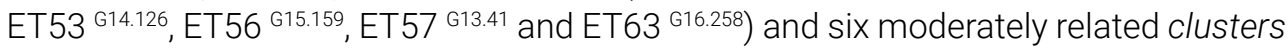
(from IX to XIV; a total of 33 isolates ${ }^{33 \%}$ or 30 ETs ${ }^{37.9 \%}$ ):

- Cluster IX: Thirteen identical and/or highly related isolates; four highly related strains (ET20 G18.15, ET61 ${ }^{613.120}$, ET66 ${ }^{65.31}$, ET70 G13.174 and G14.199).

- Cluster X: Nine highly related isolates; nine highly related strains (ET18 G13.51, ET55 G17.68, ET58 ${ }^{\mathrm{G} 17.86}$, ET59 ${ }^{\mathrm{G} 16.269}$, ET64 ${ }^{\mathrm{G} 15.131}$, ET65 ${ }^{\mathrm{G} 17.13}$, ET71 ${ }^{\mathrm{G} 15.64}$, ET73 ${ }^{\mathrm{G} 17.128}$ and ET74 ${ }^{\mathrm{G} 17.62}$ ).

- Cluster XI: Two highly related isolates; two highly related strains (ET19 ${ }^{111.13}$ and ET72 ${ }^{\text {G17.63). }}$.

- Cluster XII: Three highly related isolates; three highly related strains (ET52 G16.88, ET54 ${ }^{616.49}$ and ET69 G13.142).

- Cluster XIII: Four identical and/or highly related isolates; two highly related strains (ET62 ${ }^{617.115}$ and $\mathrm{G} 16.167$ and ET67 G11.131 and G11.32).

- Cluster XIV: Two highly related isolates; two highly related strains (ET23 ${ }^{618.91}$ and ET26 ${ }^{\mathrm{G} 18.10}$ ).

Taxon C comprised three isolates/strains (ET7 G11.96, ET17 ${ }^{612.13}$ and ET79 ${ }^{622.64}$ ) and only one moderately related cluster (XV; a total of six isolates ${ }^{6 \%}$ or five ETs ${ }^{6.3 \%}$ ):

- Cluster XV: Four identical and/or highly related isolates; three highly related strains (ET75 G17.42 andG18.124, ET78 G20.48 and ET77 ${ }^{\text {G19.44). }}$ ).

\section{Discussion}

In this study, the enzyme electrophoretic profiles of oxacillin-resistant S. aureus isolates on different gels were reproducible after three repetitions of each electrophoretic run. The discriminatory capacity (i.e., 99\% probability that two unrelated isolates sampled from a population test are classified in different strains ${ }^{\mathrm{ETs}}$ ) of the MLEE method, based on genetic interpretation of electrophoretic enzyme patterns, was also observed (i.e., the combination of existing alleles on 30 enzyme loci revealed 79 ETs). Once again, MLEE proved to be a powerful tool for the typing of $S$. aureus in epidemiological studies. These results are in agreement with previously reported data on the discriminatory power and reproducibility of the MLEE method as applied to bacteria and yeasts of medical importance ${ }^{23-27,31}$, but the discriminatory power was higher than the values reported for $S$. aureus by other groups of researchers ${ }^{29,30}$.

Genetic polymorphism has been found in almost all natural populations and at all levels of genetic organization, from genotype characteristics to phenotypic traces. The possible reasons of its existence have been the subject of a long debate in the population genetics and molecular evolution fields ${ }^{37,38}$. S. aureus is a heterogeneous species (polymorphic) ${ }^{39}$ that has been observed to have a clonal population structure ${ }^{40}$. Therefore, it is believed that $S$. aureus does not suffer extensive recombination, diversifies extensively by nucleotide mutations and displays a high degree of linkage disequilibrium (non-random asso- 
ciations between gene loci). A particular structural gene locus is defined as polymorphic when the frequency of its more common allele presents a value below 0.99 (99\%). Some of the measures used to quantify this variability in populations of organisms are the allele and gene frequencies, the percentage of polymorphic loci, the average number of alleles per locus and heterozygosity ${ }^{41}$. In this study, quantitative and qualitative variations of polymorphic loci (30 100\% polymorphic enzyme loci to one, two, three, four, five and six alleles) and the average number of alleles per polymorphic locus $(3.16 \pm 1.62)$ were observed in the population of oxacillin-resistant $S$. aureus. These variations have been observed in several studies of genetic diversity of populations of $S$. aureus obtained from human and bovine sources $29,30,42,43$. In addition, the genetic polymorphism observed in the population of oxacillin-resistant $S$. aureus isolates revealed a highly frequent polyclonal pattern and infrequent monoclonal pattern, suggesting various sources of contamination or microbial dispersion from an epidemiological point of view.

The genetic relationship between the oxacillin-resistant $S$. aureus strains was determined by using the statistic $d_{\mathrm{ij}}$ of Nei (1972) and the UPGMA dendrogram ${ }^{25,26,32,36}$, which displayed a value $r_{\mathrm{jk}}$ acceptable $\left(r_{\mathrm{jk}} \sim 0.8\right)$ based on the correlation coefficient of Pearson's product-moment [i.e., good agreement between the elements $d_{i j}$ (matrix of genetic distance) and $C_{\mathrm{jk}}$ (correlation matrix derived from UPGMA dendrogram)]. A high degree of genetic polymorphism $\left(0.000 \leq d_{i j} \leq 1.705\right)$ was observed between the ORSA isolates (i.e., on average, from zero to 170.5 allele substitutions were detected in each 100 loci from a common ancestor strain). These isolates were allotted to three taxa (A, B and C), which were distantly genetically related $\left(0.653 \leq d_{i j} \leq 1.705\right)$. Taxon A presented a larger number of isolates, strains or clusters of bacteria (60 isolates ${ }^{60 \%}, 43$ ETs ${ }^{54.4 \%}$ and eight clusters ${ }^{\text {IVIIII) }}$, followed by taxon B (33 isolates ${ }^{33 \%}, 30$ ETs ${ }^{37.9 \%}$ and six clusters ${ }^{\text {IX-XIV) }}$ ) and taxon C (six isolates ${ }^{6 \%}$, five ETs ${ }^{6.3 \%}$ and one cluster $\mathrm{xv}$ ). Each taxon presented one or more clusters and/or moderately related isolates $\left(0.282 \leq d_{i j}<0.653\right)$. In turn, these clusters harbored two or more identical and/or highly related isolates $\left(0 \leq d_{\mathrm{ij}}<0.282\right)$. Considering that highly related isolates/strains highly come from a common ancestor [i.e., descendants have suffered microevolutions and adaptations as a result of recombination (not extensive), nucleotide mutations and non-random association between gene loci (linkage disequilibrium $)^{39,40}$, these results suggest the existence of microevolutionary processes in the population of oxacillin-resistant $S$. aureus, as demonstrated in each cluster (i.e., on average, from zero to < 28.2 allele substitutions were detected in each 100 loci from a common ancestor strain). However, these data reinforce the hypothesis of the existence of several sources of contamination and/or dispersal of oxacillin-resistant $S$. aureus of clinical and epidemiologically importance, which could be associated with carriers (patients) and dental environmental (air). These epidemiological investigations have also been a goal of our research group and contribute to (i) knowledge about the dynamics of the spread and retention of $S$. aureus strains resistant to antibiotics in hospital and odontological environments (i.e., surgical devices, dental instrumentation, various surfaces, air and other) and (ii) the implementation or restructuring of containment barriers, use of personal protective equipment, means of identification and periodic treatment from professionals carriers of microorganisms (nasal cavities, oral and oropharyngeal, perineum and armpits), techniques and devices for air purification, hygiene and more efficient prophylaxis. 
Certain aspects of practicing dentistry may contribute to the transmission of microorganisms ${ }^{9}$. The skin, environment, and instruments can be contaminated with saliva, blood or organic debris during routine dental treatment ${ }^{11}$. In the dental environment, investigators have observed an increase in the amount of microorganisms during clinical procedures, suggesting contamination by aerosols, especially when high-speed handpieces or ultrasonic scalers are used ${ }^{12,13,15}$. Among the species identified in microbiological studies, Streptococcus viridans and Staphylococcus spp. are the most prevalent microorganisms found on the surfaces of dental equipment ${ }^{12-15}$. In addition, the high-speed drills and cavitrons used in dental offices generate aerosols and droplets that are contaminated with blood and bacteria and may be a route for the transmission of diseases such as SARS (severe acute respiratory syndrome), tuberculosis, and Legionnaires' disease ${ }^{44-46}$. Methicillin-resistant S. aureus (MRSA) has frequently been detected on surfaces in dental operatories, including the air-water syringe and reclining chair ${ }^{16}$. Nosocomial infections or the colonization of MRSA occurred in eight out of 140 patients who displayed no evidence of MRSA upon admission to a clinic. Antibiogram tests revealed that the isolates from the eight patients were of the same strain as those from the surfaces of the dental operatory, suggesting S. aureus transmission between the patients and the dentist via the clinical environment ${ }^{16}$. The frequency of $S$. aureus isolated from the noses, hands, and tongues of students and patients and from the clinical environment of a pediatric dentistry clinic before and after dental treatment was determined ${ }^{47}$. The highest concentration of $S$. aureus was found in the noses and on the tongues of children and among the dental students, and the highest level of contamination was observed on gloved hands, which was followed by the tongue and hands without gloves before clinical care. At the end of dental treatment, S. aureus colonies isolated from the gloved hands of students decreased significantly. Considering the clinical environment, S. aureus dissemination increased at the end of dental procedures, and the most contaminated areas were the auxiliary table and the storeroom, which was located at the center of the clinic. Such results can be explained by the intense circulation of people in the clinic and the use of high-speed dental handpieces. However, it is still speculated that much of the S. aureus contamination detected in the clinical environment came from other sources, such as direct contact, skin exfoliation or the improper handling of plates, and it is concluded that the dental clinic is an appropriate environment for $S$. aureus cross-transmission.

Because molecular-based epidemiological studies are useful in identifying possible sources of the spread of microorganisms in hospitals and dental clinical settings, this study contributes to our knowledge on the dynamics of the spread of $S$. aureus strains resistant to antibiotics and points to the need for containment barriers, use of personal protective equipment, periodic identification and treatment of carriers among clinical staff, and installation of air purifiers. Thus, infection control guidelines and published research pertinent to dental infection control principles and practices must be applied by the dentist as a matter of routine in academic dental offices. This research showed a genetic polymorphism in the population of oxacillin-resistant $S$. aureus isolates (dental patients and air of the clinical environment) and a highly frequent polyclonal pattern of these bacterial strains, supporting the hypothesis of various sources of contamination or microbial dispersion in the dental clinic environment. The isoenzyme typing and genetic relationship analysis revealed also some taxa and 
clusters exhibiting different frequencies of strains and possibly microevolutionary changes. In addition to the genetic information of $S$. aureus, the present methodology potentially collaborates with measures of prevention, management, and tracking of $S$. aureus, especially in dental clinics with great workflow.

\section{Acknowledgements}

This study was supported by the Fundação de Amparo à Pesquisa do Estado de Minas Gerais (FAPEMIG process. APQ-3897-4.03/07) and the Conselho Nacional de Desenvolvimento Científico e Tecnológico (CNPq process no. 157768/2011-2). We thank Elsevier Language Services for help with English language editing.

\section{References}

1. Lee AS, Huttner B, Harbarth S. Prevention and control of methicillin-resistant Staphylococcus aureus in acute care settings. Infect Dis Clin North Am. 2016 Dec;30(4):931-952. doi: 10.1016/j.idc.2016.07.006.

2. Loewen K, Schreiber Y, Kirlew M, Bocking N, Kelly L. Community-associated methicillin-resistant Staphylococcus aureus infection. Can Fam Physician. 2017 Jul;63(7):512-520.

3. Murashita T, Sugiki H, Kamikubo Y, Yasuda K. Surgical results for active endocarditis with prosthetic valve replacement: impact of culture-negative endocarditis on early and late outcomes. Eur J Cardiothorac Surg. 2004 Dec;26(6):1104-11.

4. Dawson MP, Smith AJ. Superbugs and the dentist: an update. Dent Update. 2006 May;33(4):198-200, 202-4, 207-8

5. Bai AD, Agarwal A, Steinberg M, Showler A, Burry L, Tomlinson GA, et al. Clinical predictors and clinical prediction rules to estimate initial patient risk for infective endocarditis in Staphylococcus aureus bacteraemia: a systematic review and meta-analysis. Clin Microbiol Infect. 2017 May 6. pii: S1198-743X(17)30236-7. doi: 10.1016/j.cmi.2017.04.025.

6. Groppo FC, Castro FM, Pacheco AB, Motta RH, Filho TR, Ramacciato JC, Florio FM, Meechan JG. Antimicrobial resistance of Staphylococcus aureus and oral streptococci strains from high-risk endocarditis patients. Gen Dent. 2005 Nov-Dec;53(6):410-3.

7. Lorber M. Endocarditis from Staphylococcus aureus. JAMA. 2005 Dec 21;294(23):2972; author reply 2972-3.

8. Motta RHL, Groppo FC, Bergamaschi CC, Ramacciato JC, Baglie S, de Mattos-Filho TR. Isolation and antimicrobial resistance of Staphylococcus aureus isolates in a dental clinic environment. Infect Control Hosp Epidemiol. 2007 Feb;28(2):185-90.

9. Autio KL, Rosen S, Reynolds NJ, Bright JS. Studies on cross-contamination in the dental clinic. J Am Dent Assoc. 1980 Mar;100(3):358-61.

10. Naeem A, Saluja SA, Krishna D, Shitanshu M, Arun S, Taseer B. Contamination of Dentist's Hands with and without Finger Rings. J Int Oral Health. 2015 Aug;7(8):114-7.

11. Suzuki J, Komatsuzawa H, Kozai K, Nagasaka N. In vitro susceptibility of Staphylococcus aureus including MRSA to four disinfectants. ASDC J Dent Child. 1997 Jul-Aug;64(4):260-3.

12. Bernardo WLC, Boriolo MFG, Gonçalves RB, Hofling JF. Staphylococcus aureus ampicillin-resistant from the odontological clinic environment. Rev Inst Med Trop Sao Paulo. 2005 Jan-Feb;47(1):19-24.

13. Motta RHL, Ramacciato JC, Groppo FC, Pacheco ABND, de Mattos-Filho TR. Environmental contamination before, during, and after dental treatment. Am J Dent. 2005 Oct;18(5):340-4. 
14. Hackney Jr. RW, Crawford MA, Tulis JJ. Using a biological indicator to detect potential source of cross-contamination in the dental operatory. J Am Dent Assoc. 1998 Nov;129(11):1567-77.

15. Smith G, Smith A. Microbial contamination of used dental handpieces. Am J Infect Control. 2014 Sep;42(9):1019-21. doi: 10.1016/j.ajic.2014.06.008.

16. Kurita H, Kurashina K, Honda T. Nosocomial transmission of methicillin-resistant Staphylococcus aureus via the surfaces of the dental operatory. Br Dent J. 2006 Sep 9;201(5):297-300; discussion 291.

17. Singh A, Goering RV, Simjee S, Foley SL, Zervos MJ. Application of Molecular Techniques to the Study of Hospital Infection. Clin Microbiol Rev. 2006 Jul;19(3):512-30.

18. Nevo E, Beiles A, Ben-Shlomo R. The evolutionary significance of genetic diversity: ecological, demographic and life history correlates. In: Evolutionary Dynamics of Genetic Diversity; 1980 p. 13-213. doi: 10.1007/978-3-642-51588-0_2.

19. Rattazzi MC, Scandalios JG, Whitt GS. Isozymes: current topics in biological and medical research. Genetic and evolution. New York: Alan R Liss; 1983.

20. Selander RK, Whittam TS. Protein polymorphism and the genetic structure of populations. In: Nei, M., Koehn, R.K. Evolution of Genes and Proteins. Sunderland: Sinuaer Associates; 1983. p. 89-114.

21. Oxford GS, Rollinson D. Protein polymorphism: adaptive and taxonomic significance. The Systematics Association Special. London: Academic Press; 1983.

22. Achtman M, Heuzenroeder M, Kusecek B, Ochman H, Caugant D, Selander RK, et al. Clonal analysis of Escherichia coli $\mathrm{O} 2: \mathrm{K} 1$ isolated from diseased humans and animals. Infect Immun. 1986 Jan;51(1):268-76

23. Napimoga MH, Kamiya RU, Rosa RT, Rosa EAR, Hofling JF, Mattos-Graner R, et al. Genotypic diversity and virulence traits of Streptococcus mutans in caries-free and caries-active individuals. J Med Microbiol. 2004 Jul;53(Pt 7):697-703.

24. Tahmourespour A, Nabinejad A, Shirian H, Rosa EA, Tahmourespour S. Typing of Streptococcus mutans strains isolated from caries free and susceptible subjects by multilocus enzyme electrophoresis. Braz J Microbiol. 2014 Jan 15;44(3):873-7.

25. Boriollo MFG, Rosa EAR, Gonçalves RB, Höfling JF. Parity among interpretation methods of MLEE patterns and disparity among clustering methods in epidemiological typing of Candida albicans. J Microbiol Methods. 2006 Mar;64(3):346-65.

26. Boriollo MFG, Dias RA, Fiorini JE, Oliveira NMS, Spolidorio DM, de Souza HM, et al. Disparity between Multilocus Enzyme Electrophoresis, Microsatellite Markers and Pulsed-Field Gel Electrophoresis in epidemiological tracking of Candida albicans. J Microbiol Methods. 2010 Sep;82(3):265-81. doi: 10.1016/j.mimet.2010.06.012.

27. Santos PO, Melo JO, Ponzzes CM, Alves JA, de Melo DL, Botelho Nde S, et al. Multilocus enzyme electrophoresis analysis and exoenzymatic activity of Candida albicans strains isolated from women with vaginal candidiasis. Mycoses. 2012 Jan;55(1):64-72. doi: 10.1111/j.1439-0507.2011.02043.x.

28. Boerlin P. Applications of multilocus enzyme electrophoresis in medical microbiology. J Microbiol Methods. 1997 Mar;28(3):221-31.

29. Fitzgerald JR, Meaney WJ, Hartigan PJ, Smyth CJ, Kapur V. Fine-structure molecular epidemiological analysis of Staphylococcus aureus recovered from cows. Epidemiol Infect. 1997 Oct;119(2):261-9.

30. Koreen L, Ramaswamy SV, Gravissm EA, Naidichm S, Musser JM, Kreiswirth BN. spa typing method for discriminating among Staphylococcus aureus isolates: implications for use of a single marker to detect genetic micro- and macrovariation. J Clin Microbiol. 2004 Feb;42(2):792-9.

31. Selander RK, Caugant DA, Ochman H, Musser JM, Gilmour MN, Whittam TS. Methods of multilocus enzyme electrophoresis for bacterial population genetics and systematics. Appl Environ Microbiol. 1986 May; $51(5): 873-84$. 
32. Soll DR. The ins and outs of DNA fingerprinting the infectious fungi. Clin Microbiol Rev. 2000 Apr;13(2):332-70.

33. van Belkum A, Struelens $M$, Visser A, Verbrugh $H$, Tibayrenc $M$. Role of genomic typing in taxonomy, evolutionary genetics, and microbial epidemiology. Clin Microbiol Rev. 2001 Jul;14(3):547-60.

34. Winn WC, Allen SD, Janda WM, Koneman EW, Schreckenberger PC, Procop GW, et al. In: Koneman's Color Atlas and Textbook of Diagnostic Microbiology. $6^{\text {th }}$ ed. Philadelphia: Lippincott Williams \& Wilkins; 2008.

35. Clinical and Laboratory Standards Institute (CLSI). Methods for Dilution Antimicrobial Susceptibility Tests for Bacteria That Grow Aerobically; Approved Standard-Ninth Edition. CLSI document M07-A9, Wayne, PA, USA; 2012.

36. Nei M. Genetic distances between populations. Am Nat. 1972;106(949):283-92.

37. Gillespie JH. The causes of molecular evolution. New York: Oxford University Press; 1991.

38. Golding B. Non-neutral evolution. New York: Chapman and Hall; 1994.

39. Fitzgerald JR, Sturdevant DE, Mackie SM, Gill SR, Musser JM. Evolutionary genomics of Staphylococcus aureus: insights into the origin of methicillin-resistant strains and the toxic shock syndrome epidemic. Proc Natl Acad Sci U S A. 2001 Jul 17;98(15):8821-6.

40. Feil EJ, Cooper JE, Grundmann H, Robinson DA, Enright MC, Berendt T, et al. How clonal is Staphylococcus aureus? J Bacteriol. 2003 Jun;185(11):3307-16.

41. Hartl DL, Clark AG. Principles of population genetics. Suderland: Sinauer Associates; 2007.

42. Musser JM, Kapur V. Clonal analysis of methicillin-resistant Staphylococcus aureus strains from intercontinental sources: association of the mec gene with divergent phylogenetic lineages implies dissemination by horizontal transfer and recombination. J Clin Microbiol. 1992 Aug;30(8):2058-63.

43. Kapur V, Sischo WM, Greer RS, Whittam TS, Musser JM. Molecular population genetic analysis of Staphylococcus aureus recovered from cows. J Clin Microbiol. 1995 Feb;33(2):376-80.

44. Harrel SK, Molinasi J. Aerosols and splatter in dentistry: a brief review of the literature and infection control implications. J Am Dent Assoc. 2004 Apr;135(4):429-37.

45. Hubar JS, Pelon W. Low-cost screening for microbial contaminants in aerosols generated in a dental office. Gen Dent. 2005 Jul-Aug;53(4):270-2.

46. Kohn WG, Collins AM, Cleveland JL, Harte JA, Eklund KJ, Malvitz DM, et al. Guidelines for infection control in dental health-care settings--2003. MMWR Recomm Rep. 2003 Dec 19;52(RR-17):1-61.

47. Negrini TC, Duque C, Oliveira ACM, Hebling J, Spolidorio LC, Spolidorio DM. Staphylococcus aureus contamination in a pediatric dental clinic. J Clin Pediatr Dent. 2009 Fall;34(1):13-8. 